\title{
Meta
}

Journal des traducteurs

Translators' Journal

\section{Lerat, Pierre (1995) : Les langues spécialisées, coll. "Linguistique nouvelle", Paris, PUF, 201 p.}

\section{François Gaudin}

Volume 41, numéro 1, mars 1996

Le(s) processus de traduction / Translation Process(es)

URI : https://id.erudit.org/iderudit/002817ar

DOI : https://doi.org/10.7202/002817ar

Aller au sommaire du numéro

Éditeur(s)

Les Presses de l'Université de Montréal

ISSN

0026-0452 (imprimé)

1492-1421 (numérique)

Découvrir la revue

Citer ce compte rendu

Gaudin, F. (1996). Compte rendu de [Lerat, Pierre (1995) : Les langues spécialisées, coll. "Linguistique nouvelle", Paris, PUF, 201 p.] Meta, 41(1),

172-174. https://doi.org/10.7202/002817ar

Ce document est protégé par la loi sur le droit d'auteur. L'utilisation des services d'Érudit (y compris la reproduction) est assujettie à sa politique d'utilisation que vous pouvez consulter en ligne.

https://apropos.erudit.org/fr/usagers/politique-dutilisation/
Cet article est diffusé et préservé par Érudit.

Érudit est un consortium interuniversitaire sans but lucratif composé de l’Université de Montréal, l'Université Laval et l'Université du Québec à Montréal. Il a pour mission la promotion et la valorisation de la recherche. https://www.erudit.org/fr/ 
LERAT, Pierre (1995) : Les langues spécialisées, coll. «Linguistique nouvelle», Paris, PUF, $201 \mathrm{p}$.

«La matière de ce livre est une réflexion globale sur les 'langues de spécialité'», annonce l'auteur au seuil de son livre. Fidèle à son style, dont la précision et la concision ne sont jamais prises en défaut, Pierre Lerat dresse, en moins de 200 pages, un large panorama sur les langues spécialisées, balayant leur horizon en dix-sept chapitres. Le premier, «linguistique, langue, terminologie, langue spécialisée», dessine la perspective d'ensemble, les autres étant consacrés aux fondements linguistiques des langues spécialisées (linguistique générale, systèmes de signes, les unités terminologiques, écriture, morphologie, syntaxe, sémantique), à leurs applications (traduction, documentation, normalisation, aménagement linguistique, rédaction, ingénierie de la connaissance, lexicographie, terminographie), et à leur place dans l'enseignement.

La matière annoncée est riche, et c'est à partir d'elle que l'auteur affirme sa volonté de rédiger là un «livre de professeur». De fait, l'ouvrage est clair et rigoureux, au meilleur sens du terme, soucieux d'examiner les prémisses mêmes de la pratique et de la théorie de ces «langues spécialisées» et d'en étudier les contours et le fonctionnement à la lumière d'une solide culture linguistique.

Cet examen attentif de la cohérence de la doctrine se traduit tout d'abord dans le choix du titre. Aux yeux de l'auteur, la notion usuelle de «langues de spécialité» est contre-intuitive, il lui préfère donc celle de «langues spécialisées». Le bénéfice de la dénomination choisie tient à «la souplesse des interprétations: il y a place pour des degrés variables de spécialisation». Elle permet de construire une notion pragmatique, renvoyant à «une langue naturelle considérée en tant que vecteur de connaissances spécialisées» (p. 20). De ce fait, la définition, purement fonctionnelle, des langues spécialisées autorise une variété dans la technicité des formulations, les connaissances qui motivent leur emploi étant désignées de façon conventionnelle par des termes.

Ayant ainsi clarifié le terrain, l'auteur inclut toute théorie des langues spécialisées au sein d'une «théorie générale des langues». Cette dernière suppose une culture linguistique variée et ouverte sans exclusive sur les univers théoriques pertinents. Elle serait nécessaire aux experts langagiers qui évalueraient les initiatives linguistiques du législateur, l'exemple pris de la féminisation des noms de métiers pouvant être élargi aux arrêtés terminologiques, si contestés à l'occasion du débat sur la loi Toubon. On remarquera tout de même que l'auteur adopte ici sur une conception de la linguistique, à laquelle les avancées de la sociolinguistique seraient étrangères...

La sémiotique fournit le cadre englobant de la formation linguistique dont l'ouvrage dessine les contours : leur morphologie l'atteste, la formation des termes ne se limite pas au seul système linguistique. La pluralité des codes, l'internationalisation des échanges, les standardisations consenties contribuent à la complexité des langues spécialisées. Leur étude impose donc une approche sémiotique plurielle.

Au premier rang figure l'étude des terminologies et l'auteur rappelle l'intérêt des critères conceptuels face à l'opérativité limitée des critères lexicologiques pour délimiter les unités terminologiques. Les faisceaux d'indices linguistiques confirment la pertinence des séries morphologiques, des cooccurrences et des relations de dépendance.

Peu sensible à la pureté sémiotique de l'oral, Pierre Lerat tient que «la connaissance des pratiques passe par l'écrit au sens large» (p. 56). Ce point de vue, légitime dans un souci de pragmatisme, risque d'occulter pour le lecteur non averti tout un pan de l'étude des terminologies, celui de leur genèse, en même temps qu'il implique que l'on délaisse, dans l'étude des langues spécialisées, leur mode d'existence majoritaire dans les pratiques langagières. Il est vrai que la seule question de la typologie des textes spécia- 
lisés soulève des problèmes considérables, et même l'énumération à la Prévert citée de Kocourek laisse apparaître des lacunes. Si l'on parle beaucoup à l'occasion des pratiques professionnelles, l'écrit omniprésent remplit des fonctions très variées.

La position de l'auteur est donc celle d'un réaliste. Les études sur l'oral restent marginales et seraient à encourager. Mais, en l'état actuel, le terminologue est un homme de l'écrit, voire de bureau. Qu'il travaille à la représentation des connaissances, en aménagement de la langue, ou à la traduction spécialisée, il va de documents en documents et doit donc résoudre tous les problèmes liés à l'écrit.

Le chapitre sur la morphologie intéressera le lecteur principalement pour le développement relatif aux paradigmes dérivationnels, repris de Louis Guilbert, lesquels permettent de constituer des séries lexicales, sous réserve de régler l'épineuse question des allomorphismes et des supplétions. Il reste à leur consacrer une étude large afin de tester leur pertinence dans une orientation de diagnostic linguistique.

Les relations entre syntaxe et langue spécialisée sont évoquées très rapidement, au motif qu'en la matière, on attend du langagier la seule «aptitude à rendre compte linguistiquement d'habitudes d'expression statistiquement dominantes dans tel type de texte» (p. 75). Les relations de dépendance, logiques et énonciatives, tiennent une place centrale dans la description. Ce qui est ici esquissé suscite l'intérêt car la syntagmatique, qu'elle soit de l'écrit ou de l'oral, s'avère de première importance pour les progrès de la connaissance dans ce domaine d'étude, mais le lecteur intéressé, fût-il néophyte, risque de rester sur sa faim.

La sémantique constitue un terrain de choix pour l'auteur, signataire d'une Sémantique descriptive. La sémantique qu'il privilégie ici est essentiellement relationnelle : sont passées en revue les relations lexicales, morphologiques, syntagmatiques, de dépendance, logiques et énonciatives, ces dernières renvoyant surtout à la fonction dénotative qui fonde l'effort de monoréférentialité dans l'usage des termes. Bien sûr, il ne faut confondre ici référent et objet. La référence, source de difficultés et de débats innombrables, est chez l'auteur cette fonction identifiante variable selon les groupes de locuteurs ( $c f$. p. 45 ). On peut voir là un indice de la nécessité d'une sociosémantique : puisque «la sémantique des connaissances n'est pas la fin de la science, mais son appréhension linguistique», la pluralité des groupes de locuteurs implique de restaurer sa dimension de variation au sens que les locuteurs donnent au monde par leurs mots.

La sémantique se retrouve dans l'approche que fait l'auteur de la traduction. Puisant dans ses travaux antérieurs, de Molière au droit comparé, il indique des pistes pour une approche plus linguistique des problèmes de traduction, réservant une place de choix à la prise en compte des collocations, bien démarquées des cooccurrences.

La proximité entre terminologie et documentation met en relief à quel point cette dernière souffre d'une approche trop pauvre des relations entre mots clés. Or analyse des notions et analyse des dénominations sont également indispensables et le rapprochement des deux disciplines devrait conduire à une amélioration de l'efficacité des consultations.

Normalisation et aménagement linguistique ont en partage un désir de pouvoir sur la gestion des dénominations. Leurs motivations les distinguent; leurs moyens les réunissent souvent. La normalisation industrielle répond à des besoins économiques. Mais le gouvernement de «l'empire des normes» (p. 115) suppose une autorité qui s'impose à tous, d'où le rôle des États en la matière. Il existe donc une dimension de politique linguistique dans les deux activités. Comme le rappelle Pierre Lerat, «l'intervention du politique sur les langues est universelle» (p. 127). Mais les attitudes et idéologies linguistiques sont variées et les façons d'agir doivent en tenir compte. Il importe notamment de «se donner des moyens significatifs pour rendre effective la politique d'aménagement du corpus grâce à des actions d'implantation terminologique» (p. 133). 
La rédaction technique rejoint la traduction par la place qu'y joue la reformulation, gage de compréhension de documents de départ. Ainsi, si «l'ésotérisme est une menace qui pèse constamment sur la communication spécialisée» (p. 138), c'est qu'il résulte souvent d'une compréhension dont la formulation reste cantonnée à des réseaux linguistiques limités. Ici, la démarche purement onomasiologique doit se doubler d'une labilité verbale plus que d'une recherche d'univocité. Par certains aspects, elle se rapproche de la vulgarisation : plus que rédiger vrai, il faut rédiger juste. Pour améliorer la rédaction, il faut donc aider à la reformulation dans le respect de la pertinence, «adéquation à la matière considérée» (p. 143), et de la convenance «appropriation au type de texte» (p. 144). Ici les collocations jouent un rôle essentiel pour une expression «ajustée à des habitudes d'expression» (p. 145). Leur étude renvoie à une stylistique spécialisée.

Le chapitre consacré à l'ingénierie de la connaissance se fonde sur une approche purement propositionnelle de la connaissance, répartie en domaines structurables. On voit ici l'influence d'une méthodologie onomasiologique, celle de la terminologie, basée non sur des classes de langue (comme ce serait le cas en sémantique avec des taxèmes) mais sur des découpages documentaires. Il reste que l'ingénierie de la connaissance ouvre une avenue au progrès de la connaissance et de la description, notamment en «responsabilisant les sémanticiens» (p. 152). La revue des travaux en traitement automatique se conclut sur une proposition de bases unifiant la terminographie et la lexicographie.

Cette dernière se voit examinée à la lumière de l'opposition, illustrée par l'examen du traitement d'angine dans trois grands dictionnaires, entre sémantiques extrinsèque et intrinsèque des termes/mots. Les deux niveaux sémiotiques renvoient à l'éternel dilemme : polysémie ou homonymie? La recherche de sa solution est pour l'auteur l'occasion d'une «utopie» (p. 167) : l'application du programme du DFC à une lexicographie terminologique multidomaines. C'est poser l'ambitieux programme d'un «dictionnaire historique encyclopédique des sciences et techniques» qui soit vraiment un dictionnaire et vraiment une encyclopédie. Et historique, car seule l'histoire permettra de relier, en une histoire des discours, l'histoire des mots et celle des idées. Des esquisses d'une telle démarche sont éparpillées dans la littérature de vulgarisation, mais la difficulté principale réside dans l'insertion effective de cultures spécialisées dans une culture englobante.

Pour sa part, la terminographie est vouée à une description linguistique aussi fine que possible du fonctionnement des noms de notions. Sa méthodologie impose un strict traitement synchronique. Loin des soucis culturels, pragmatisme et efficacité priment. L'auteur la préfère multifonctionnelle et inscrite dans la «tradition de la grande lexicographie» (p. 178), elle doit alors viser des usages multiples en étant objective et systématique. Mais l'ambition des projets est freinée par la circulation des données et la possibilité de dépouillement terminologique assisté. Si la propriété intellectuelle et le droit au pessimisme alimentent les doutes de l'auteur, il dessine pour l'avenir une place de choix pour les linguistes dans une lexicographie revivifiée par la terminographie.

La conclusion est un plaidoyer argumenté pour un enseignement des langues spécialisées : alphabétisation technique et scientifique, enseignement des langues vivantes et de la traduction spécialisée, ouverture aux divers systèmes sémiotiques et à une syntaxe pluridimensionnelle. Mais aussi, l'auteur souhaite voir une professionnalisation de l'aménagement linguistique, ce qui est un exemple des places que notre professeur aimerait voir reconnues aux «linguistes qualifiés» de demain. À charge pour ces derniers de lire aujourd'hui ce livre utile, qui vient combler une lacune dans l'approche linguistique des langues spécialisées et dans lequel ils trouveront une bibliographie complète et très à jour. 